\title{
Evaluation of electromechanical impedance based structural health monitoring for detection of loosening in total knee arthroplasty
}

\author{
Robert I. Ponder ${ }^{1}$, R. Michael Meneghini ${ }^{2}$, Steven R. Anton ${ }^{1 *}$ \\ Dynamic and Smart Systems Laboratory ${ }^{1}$ \\ Department of Mechanical Engineering, Tennessee Tech. University, 115 W. 10th St., Cookeville, \\ TN 38505 \\ Indiana University School of Medicine ${ }^{2}$ \\ Department of Orthopedic Surgery, 550 N. University Blvd., Suite 6201, Indianapolis, \\ IN 46202
}

\begin{abstract}
Total Knee Arthroplasty (TKA) continues to be a common and important orthopedic procedure for many in the United States. Despite recent medical advancements and increasing knowledge in the orthopedic community, it has been determined that $20 \%$ of TKA patients are still dissatisfied with their knee replacements. Causes of this failure include septic loosening and wear on the bearing component of the implant. Another cause of failure that has received specific attention from the mechanical community is aseptic loosening, which has been attributed to unbalanced ligaments or misalignment of the implant components. Previous efforts have been made to detect loosening by using passive force sensors such as piezoelectric transducers or strain gauges to detect misalignment. An alternative to this is to perform active sensing or structural health monitoring to evaluate possible loosening before it becomes a critical concern to the patient. One method of structural health monitoring, called the electromechanical impedance (EMI) method, is particularly attractive as it can use a single, compact piezoelectric transducer to determine the state of the host structure. This work is intended to evaluate the ability of the EMI method in sensing loosening between the cement and bone of a TKA tibial tray. This work will utilize real tibial trays implanted into synthetic bone (Sawbone) to evaluate the feasibility of detecting loosening using the EMI method. The intention of this work is to serve as a foundation for further in-vivo and intraoperative studies.
\end{abstract}

Keywords: structural health monitoring, piezoelectric, impedance, total knee replacement, loosening, biomedical

\section{INTRODUCTION}

As the American population continues to age, the demand for total knee replacements (TKR) progressively increases as well ${ }^{1}$. This demand has created a push to improve patient outcomes from TKR procedures. Currently, about a fifth of all TKR patients are dissatisfied with their procedural outcomes ${ }^{2,3}$. Further, approximately $8 \%$ of all total knee replacements will fail within 10 years post-operative ${ }^{4}$. Though implants can fail in a variety of ways, the most common modes are infection and loosening. When investigating failure due to loosening, it is often found that the failure occurs at the cemented interface between the tibial component and the tibia bone, both shown in Figure $1^{5,6}$. Because of the mechanical nature of failure due to loosening, there has been significant drive to further understand the mechanical conditions that lead to or predict such a failure. Historically, the mechanical conditions in the TKR system have been investigated using force plates and fluoroscopy ${ }^{7}$. Recently, the development of smaller sensing technology has given way to embedding sensors inside of the patient's implant, allowing for more direct force and pressure distribution measurements ${ }^{8}$. One method that has shown promise as a force sensing technique is embedding piezoelectric transducers between the tibial tray and bearing component of the implant ${ }^{9}$. Piezoelectric transducers benefit from being able to perform both energy

* santon@tntech.edu; phone 1931 372-3287; fax 1931 372-6340; blogs.cae.tntech.edu/santon

Health Monitoring of Structural and Biological Systems XIII, edited by Paul Fromme,

Zhongqing Su, Proc. of SPIE Vol. 10972, 1097226 · C 2019 SPIE

CCC code: $0277-786 \mathrm{X} / 19 / \$ 18 \cdot$ doi: $10.1117 / 12.2514053$ 


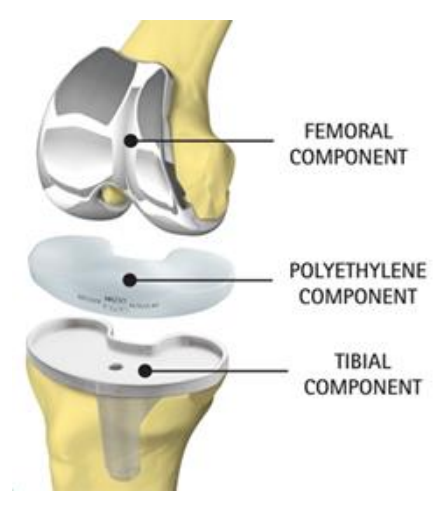

Figure 1 Components of a Total Knee Replacement (TKR).

harvesting and force sensing function, creating the possibility for a self-contained, self-powered force sensing system ${ }^{10}$. It should be noted that piezoelectric transducers are often referred to as PZT's, due to the common piezoelectric material lead zirconate titanate.

In addition to performing energy harvesting and force sensing, piezoelectric transducers also possess the ability to perform certain forms of structural health monitoring (SHM) ${ }^{11}$. SHM would benefit the TKR community as it would allow detection of changes in the mechanical conditions of a TKR implant, prior to it becoming a hazard for the patient. This could reduce the need for patients to receive multiple costly post-operative x-rays that are normally required to detect loosening. Methods of SHM in which piezoelectric transducers are used include guided wave and the electromechanical impedance (EMI) method ${ }^{12}$. Between these two methods, the EMI method is particularly interesting as the associated measurement system has the potential to maintain a compact profile. This is particularly useful in the space-limited area of a TKR implant. The EMI method has already been heavily researched in the fields of aerospace and civil structures ${ }^{13}$. With this said, little research has been done on the medical applications for SHM, with an even smaller amount of research on EMI specific applications. One study which serves as an exception was intending to investigate the application of EMI based SHM in detecting loosening of dental implants ${ }^{14}$. The success found in determining the stability of dental implants may suggest the ability to detect stability in orthopedic implants. Additionally, another study from the Dynamic and Smart Systems Lab at Tennessee Technological University displayed the ability to use the EMI method to detect damage in a geometrically generic cemented implant-bone interface ${ }^{15}$. This study successfully showed that the EMI method has the potential to detect damage in a cemented interface with materials found in the TKR system. With this said, it did not account for specific geometries that occur at the interface between the tibial component and tibial bone.

From the current literature, it is apparent that only preliminary studies into the use of EMI based SHM in total knee replacements have been investigated. What is needed is a study of the effectiveness of the EMI method on an actual knee implant. This study intends to fill that need by extending the author's previous work and investigating damage detection of loosening of the cemented interface between an actual tibial component and a simulated tibia bone. Specific focus is given to loosening that occurs as a result of separation between the tibial cement and the underlying bone structure, as this failure mode has been shown to be common ${ }^{6}$. In this study, test samples for a healthy implant system as well as weakened and failed implant systems are created. Measurements are taken in the frequency domain using the EMI method and compared in order to determine if there is a detectable difference between the healthy, weakened, and failed conditions. Additionally, an algorithm is developed to map key frequency bandwidths which consistently show differences between the healthy implant system and the weakened and failed implant systems. Finally, example bandwidths are selected and overlaid with the original data to evaluate which measurement features show impactful change between the healthy implant system and the weakened and failed implant systems.

This study is intended to provide a basic view of the EMI method's ability to detect loosening in total knee replacements. The knowledge gained from this study includes a better understanding of the impedance response of the TKR system as well as an understanding of the effectiveness of the EMI method with an actual tibial component. The long-term goal is to use the knowledge gained from this study to perform cadaver or in vivo testing of loosening in the future. The final objective is to implement SHM in patient implants, ideally predicting loosening failure before it becomes a health hazard. 


\section{METHODOLOGY}

\subsection{Electromechanical impedance method}

The electromechanical impedance method of SHM was first developed in 1994 and remains a relatively simplistic method of structural health monitoring ${ }^{16}$. The guiding principal of the EMI method is centered around the electrical and mechanical coupling of piezoelectric materials. In the EMI method, a piezoelectric transducer is adhered to the host structure that will be monitored, in this case the implant system. When the piezoelectric is adhered to the structure, the mechanical impedance of the system becomes a combination of the mechanical impedance of the PZT and the structure. Due to the coupled nature of the PZT, changes in the mechanical impedance cause a measurable change of the electrical impedance. Further, if the mechanical impedance of either the PZT or the host structure changes, this has the potential to show up as a measurable change in electrical impedance. Damage can be detected using the EMI method by measuring the difference between the electrical impedance of a damaged and healthy structure. Historically, impedance methods have used large and cumbersome impedance analyzers to perform measurements. However, in recent years, the potential for using smaller data acquisition hardware has made it conceivable that a smaller measurement device could be used to effectively detect damage ${ }^{17}$. This suggests the potential for the EMI method to be used to detect damage in a physically constrained environment such as a TKR.

\subsection{Creation of test samples}

As previously stated, the presented work will investigate three different conditions pertinent to the detection of loosening between the tibial component and tibia. The three conditions will include a healthy implant system, a weakened implant system, and a failed implant system. The healthy implant system is intended to represent a tibial component that is properly cemented into the tibia bone structure. The weakened implant system is intended to represent a tibial component that is not properly cemented into the tibia bone structure but has not yet failed. Finally, the failed implant system represents a tibial implant that has become loose to the point that it can no longer safely or properly function and presents a health hazard to the patient. Test samples representing these three conditions must be created and measured for comparison.

In order to replicate the implant system, representation of the TKR's tibial component, bone cement, and tibia bone structure is needed. For the tibial component, a size 5 DJO Empower 3D tibial tray is used. The bone cement selected is Stryker VeraPlex HV bone cement (Stryker Corporation). This bone cement relies on a dry powder and liquid monomer which must be mixed before it hardens. For the tibia bone structure, a foam-based artificial bone, referred to as Sawbone, is used (Sawbones USA). This Sawbone has a cross section that is similar in shape to a tibial cross section (Model \#1522912). Additionally, the Sawbone has a dense outer shell with a porous core made of 40 PCF and 12.5 PCF Sawbone material, respectively. These two densities are intended to represent the dense outside and porous inside of an actual tibia bone. Figure 2 displays images of the tibial component and Sawbone used in this work.

The process for creating the test samples of the three conditions is similar to the actual implantation process for the tibial tray component of a TKR. Because of this similarity to the actual surgery, a board-certified orthopedic surgeon (Dr. R. Michael Meneghini, co-author) created all test samples. For the healthy test samples, the two components of the cement mixture are mixed together for 6 minutes in order to gain a desired consistency. During this mixing process, an orthopedic surgical tool referred to as a "tibial punch" is used to create a hole in the top of the Sawbone that is roughly in the shape

(a)

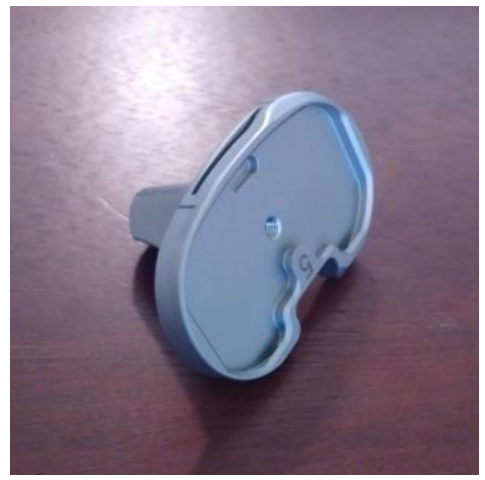

(b)

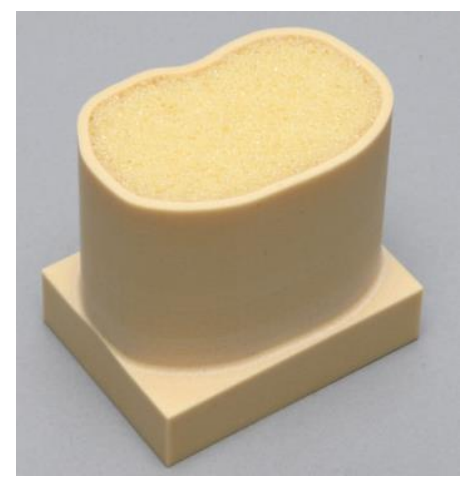

Figure 2. Image of a) DJO Empower 3D tibial tray and b) the selected Sawbone structure. 
of the stem of the tibial tray. Once the bone cement is mixed, it is applied to the bottom of the tibial tray and the top of the Sawbone. The tibial tray is then placed inside of the hole created by the tibial punch. A standard hardware hammer is then used to strike the top of the tibial component in order to seat it into the Sawbone. Once the tibial component is firmly seated inside the Sawbone, the cement is allowed to harden. In order to verify repeatability of experimentation, seven healthy implant samples are created. Since access to only one DJO tibial tray was available for experimental testing, the current sample must be tested and then destroyed in order to recover the tibial tray for the next sample.

The process for simulating the weakened implant environment is similar to that of the process for creating a healthy implant setup. The exception is that before the application of any cement, a coating of mineral oil is applied to the Sawbone. As a result of this layer of mineral oil, the bone cement firmly adheres to the tibial component but does not adhere as well to the Sawbone. This lack of adhesion to the Sawbone is due to a lack of chemical bonding between the cement and the bone as well as a reduced ability for the cement to seep into the pores of the inner bone structure in the presence of the mineral oil. This poor adhesion to the porous bone structure has been hypothesized to be a cause of tibial loosening in past clinical studies $^{6}$. A total of six weakened samples are created once again for repeatability. After the weakened measurements are taken, the weakened cement is separated from the porous Sawbone using a small knife inserted between the cement and the Sawbone. This new separated state is representative of a knee implant which has failed due to loosening. Measurements are then taken for this failed implant condition. It should be noted that both weakened and failed measurements are taken prior to moving on to the next sample. As a result, there are six failed sample measurements to complement the six weakened sample measurements.

\subsection{Experimental setup}

Impedance measurements of the test samples are made in the frequency domain using an HP 4194A impedance analyzer (Hewlett Packard). A small PZT is adhered to the top of the tibial component using super glue and connected via electrical leads to the impedance analyzer. The PZT is a $12.85 \mathrm{~mm}$ diameter, $2.1 \mathrm{~mm}$ thick piezoceramic made from APC $850 \mathrm{PZT}$ material (APC International, Ltd). The PZT is adhered $14 \mathrm{~mm}$ from the anterior edge of the tibial tray and $10 \mathrm{~mm}$ from the centerline, as displayed in Figure 3. Tabbed electrodes on the top of the piezoelectric allow for easy electrical access to the surface that is directly adhered to the tibial component. It should be noted that due to the destructive nature of removing the tibial component from a sample after a measurement as well as the hammering of the tibial component during the implanting process, a new piezoelectric is used for each new healthy and unhealthy test sample. A new PZT is not placed on the tibial component between the weakened and failed measurements as no destructive process is required to create the failed state. The test samples are clamped to a table using a table vice in order to provide a consistent fixed end condition. This end condition also provides consistency with previous studies ${ }^{15,18}$.

As previously stated, seven healthy implant samples and six unhealthy implant samples are created and tested. The unhealthy samples are tested in both a weakened and failed state. Measurements are taken within a frequency range of 10 $\mathrm{kHz}$ to $310 \mathrm{kHz}$, at a measurement resolution of $25 \mathrm{~Hz}$. Data is imported from the impedance analyzer into a computer running a LabVIEW interface via a GPIB-to-USB interface cable. This collected data is then stored in a text file and imported into MATLAB for data processing.

(a)

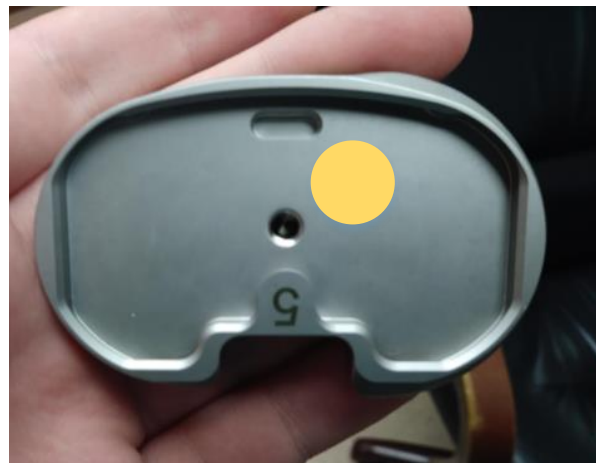

(b)

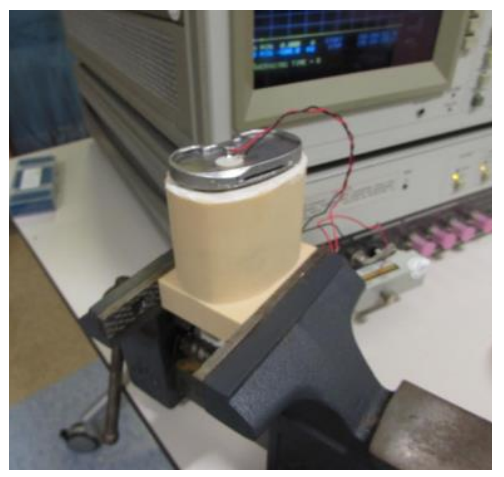

Figure 3. Image of a) the approximate location of the PZT transducer and b) a completed healthy implant test setup in the experimental configuration. 


\subsection{Data processing algorithm}

Due to the size of the data set and the complexity of the response signals, it is desirable to develop an algorithm which conveys what areas and features of the frequency response are sensitive to the weakened and failed knee conditions when compared to the healthy implant system. The algorithm should be able to take the raw data samples for the healthy knee (baseline) and the unhealthy condition (damaged) and return a value of statistical significance between the two states. The raw data evaluated should be impedance measured across the frequency domain. The real component of impedance is evaluated as it has been shown to be the most sensitive component ${ }^{19}$. The presented algorithm designed for this work will return a value of statistical confidence that shows how certain the differences in measured impedance is caused by a measurable change rather than experimental error or natural variation between samples. The presented algorithm performs three operations in order to go from the raw data to statistical confidence: feature selection, evaluation via a damage metric, and calculation of statistical confidence. The end values can then be mapped across various measured features in order to optimize the resulting statistical confidence.

The first component of the algorithm is feature selection. It is well known in impedance-based SHM that certain features of the impedance spectrum will be more sensitive to damage than others, therefore, it is common practice to extract only these sensitive bandwidths for inclusion in a damage metric algorithm. Since it is uncertain whether the peaks in measured impedance or the areas between peaks on the frequency spectrum show the most statistically significant difference, it is desirable to sweep the entire measured spectrum, evaluating various bandwidth sizes for inclusion in the damage metric algorithm. This means evaluating individual peaks as well as multiple peaks grouped together. During the mapping of the algorithmic results, it is best to map statistical confidence off location in the measured spectrum, from $10 \mathrm{kHz}$ to $310 \mathrm{kHz}$. Additionally, the map should display results centered at a given frequency across all possible bandwidth sizes that do not exceed the limits of the measured spectrum. This means that the selected features are all possible measured frequencies, each of which is evaluated across all possible bandwidths at that frequency.

The second component of the data processing algorithm is evaluation via a damage metric. For this study the selected damage metric is root-mean-square-difference (RMSD). RMSD has been shown to be a simplistic yet effective method for quantifying the difference between signals across a given bandwidth ${ }^{20}$. RMSD is calculated as

$$
\operatorname{RMSD}=\sqrt{\frac{\sum_{i=1}^{n}\left(z_{i, u}-Z_{i, h}\right)^{2}}{n}},
$$

where $n$ is the number of points in the bandwidth, $i$ is an index, and $Z_{h}$ and $Z_{u}$ are the signals for the healthy and unhealthy conditions, respectively. Naturally, the RMSD equation requires the calculation of a difference between two signals. For this algorithm, the difference is taken between a given sample's impedance response and the average response of all seven healthy implant systems. This calculation is done for each unhealthy and healthy sample being compared. As a result, for a given frequency and bandwidth selected in the previous step, there are seven healthy RMSD values and twelve unhealthy RMSD values, six for the weakened implant and six for the failed implant. These are then statistically compared in the final component of the algorithm.

The last component of the algorithm is the calculation of statistical significance. This component involves evaluating the previously derived RMSD values to determine if there is a difference between the healthy and unhealthy conditions. For this part, the t-test is used as it performs well with small sample sizes. Welch's version of the t-test is used as it cannot be assumed that the variation of the healthy and unhealthy RMSD values is equal. The resulting t-score from the t-test is converted using MATLAB's internal equations and tables to find a statistical confidence. The equation for Welch's t-test is given as

$$
\mathrm{t}-\text { score }=\frac{\bar{X}_{u}-\bar{X}_{h}}{\sqrt{\frac{s_{u}^{2}}{n_{u}}+\frac{s_{h}^{2}}{n_{h}}}},
$$

where $\bar{X}_{h}$ and $\bar{X}_{u}$ are the averages of the RMSD values for the healthy and unhealthy conditions, respectively, $s_{h}$ and $s_{u}$ are the sample standard deviations for the healthy and unhealthy condition RMSD values, respectively, and $n_{h}$ and $n_{u}$ are the number of samples for the healthy and unhealthy conditions, respectively. 
As mentioned before, the resulting confidence values are mapped across frequency and bandwidth size. The weights of the various mapped regions are assigned based off the confidence calculated from the algorithm for the given region. Two separate maps are to be created, one comparing healthy and weakened implant responses and the other comparing healthy and failed implant responses. It should be noted that the medical community typically uses a confidence of $95 \%$ as the point by which statistical significance is achieved. As a result, the created maps focus on regions that have confidences higher than $95 \%$. The developed maps should provide insight to where and to what extent the impedance responses of the healthy and unhealthy implant systems differ.

\section{RESULTS AND DISCUSSION}

\subsection{Qualitative analysis of healthy and unhealthy conditions}

Before quantitatively evaluating the collected data for the unhealthy and healthy conditions, it is useful to qualitatively inspect the features of the impedance responses to look for key patterns. As a result, this section displays a comparison between the healthy and weakened conditions as well as the healthy and failed conditions. First, Figure 4 displays an overlay of the healthy implant impedance responses and the weakened implant impedance responses. Looking at the overall trend of the implant responses, it is apparent that significant activity occurs between $10 \mathrm{kHz}$ and $50 \mathrm{kHz}$ for the weakened samples as well as between $200 \mathrm{kHz}$ and $270 \mathrm{kHz}$ for both weakened and healthy samples. Between these two regions, the response between 10 and $50 \mathrm{kHz}$ shows much more consistent and well-defined features such as peaks. The first peak detected, which is at approximately $11 \mathrm{kHz}$, shows an exceptionally large amplitude compared to other peaks in the region. Looking at the weakened implant data in the same figure, it is apparent that the response shows the same general trend as the healthy implant data. Looking at the active region between 200 and $270 \mathrm{kHz}$, it is difficult to make any qualitative deductions, due to the variation of the features and lack of apparent difference between the healthy and weakened implant systems. At the lower active region between 10 and $50 \mathrm{kHz}$, there are multiple apparent differences between the healthy and weakened implant systems. The first peak at $11 \mathrm{kHz}$ experiences a frequency shift between the two conditions as well as an increase in impedance for the weakened condition. Additionally, the peaks at 20, 26, and 31 $\mathrm{kHz}$ for the weakened condition are much more apparent than the same features in the healthy implant. It should be noted that there is some additional new activity in the weakened implant samples between 60 and $80 \mathrm{kHz}$ but it appears to be isolated to only one weakened sample.

In addition to the weakened implant samples, the failed implant samples are cross examined against the same baselines. The results for the failed implant samples overlaying the healthy implant samples are displayed in Figure 5. It is immediately apparent that there is significantly more activity in the failed condition than in the weakened condition. Overall the response of the failed condition still follows the same general trend but is not as smooth as the weakened or

(a)

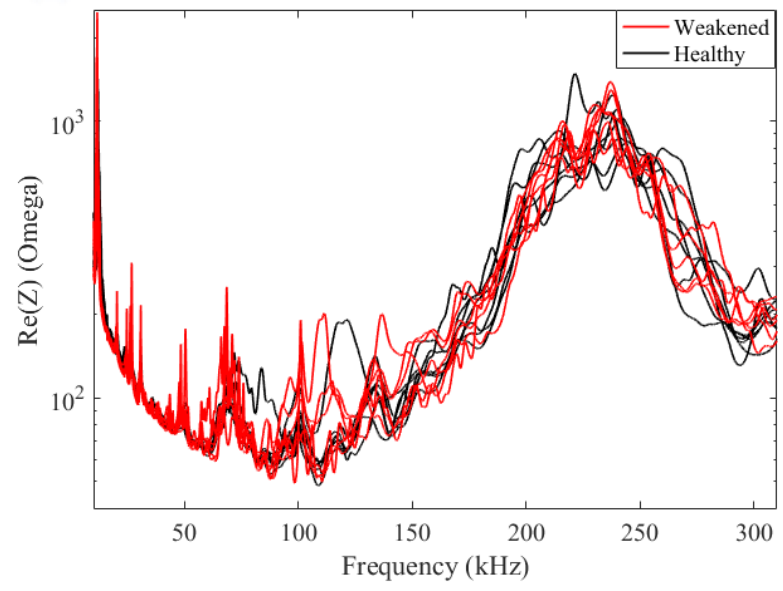

(b)

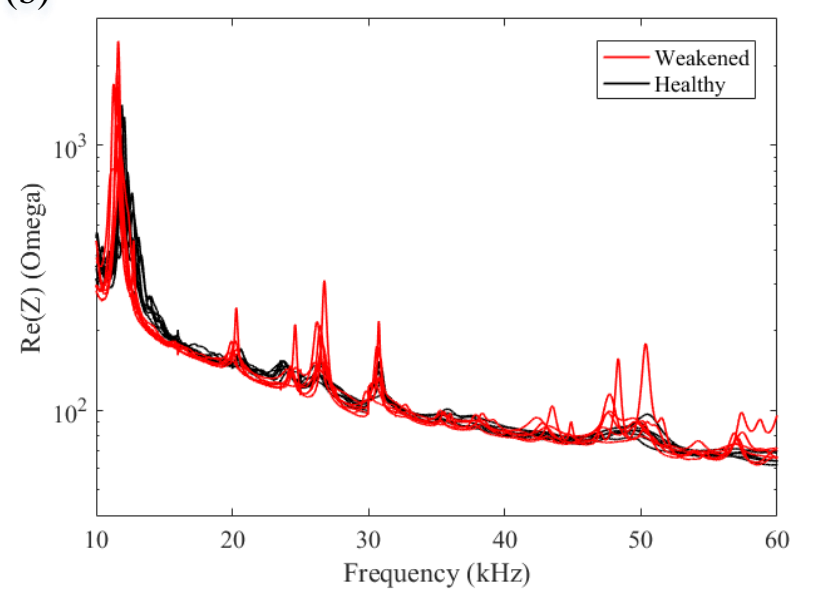

Figure 4. Measured impedance spectrum of the weakened and healthy test samples across a) $10 \mathrm{kHz}$ to $310 \mathrm{kHz}$ and b) $10 \mathrm{kHz}$ to $60 \mathrm{kHz}$. 
(a)

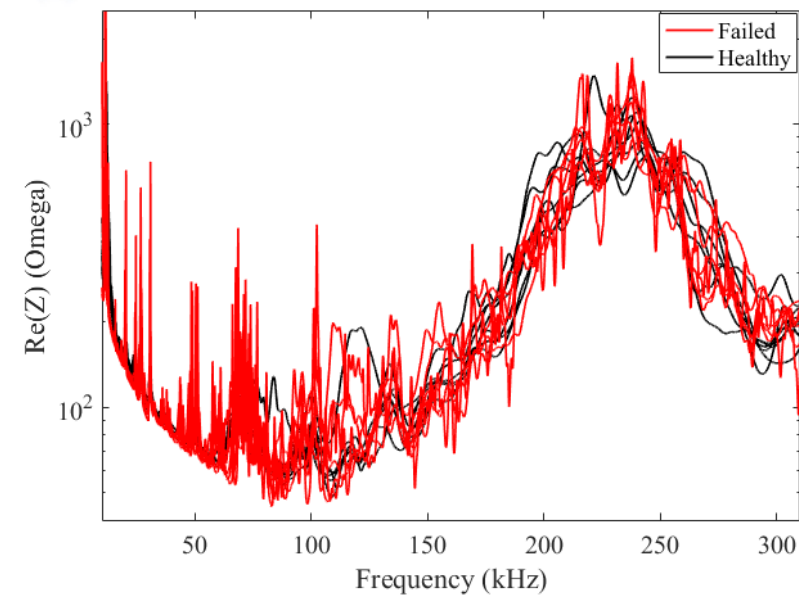

(b)

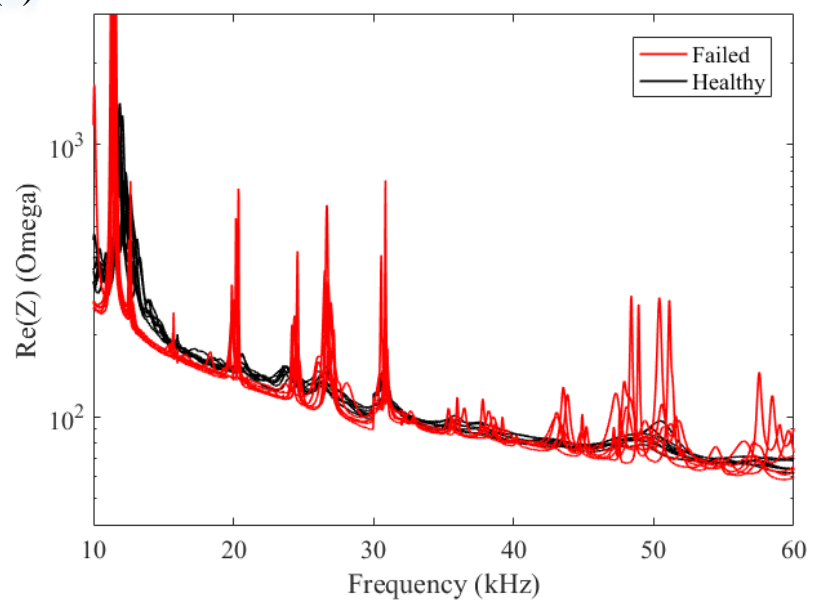

Figure 5. Measured impedance spectrum of the failed and healthy test samples across a) $10 \mathrm{kHz}$ to $310 \mathrm{kHz}$ and b) $10 \mathrm{kHz}$ to 60 $\mathrm{kHz}$.

healthy implants, even in areas of low activity. The peaks at 20, 26, and $31 \mathrm{kHz}$ have grown even more different from the healthy implant than what was displayed in the weakened condition. Additionally, more activity has occurred between 50 $\mathrm{kHz}$ and $80 \mathrm{kHz}$ than the healthy and weakened conditions. The peak at $11 \mathrm{kHz}$ maintains a similar frequency shift to what was seen in the weakened condition. This qualitative difference between the weakened and failed condition is promising as it may suggest the ability to determine degradation in mechanical stability as a patient's implant loosens.

\subsection{Quantitative results from mapping algorithm}

The data presented in the previous section is quantitatively evaluated using the algorithm discussed in the methodology section of this paper. Figure 6 displays the resulting map from the six healthy and seven unhealthy test samples when

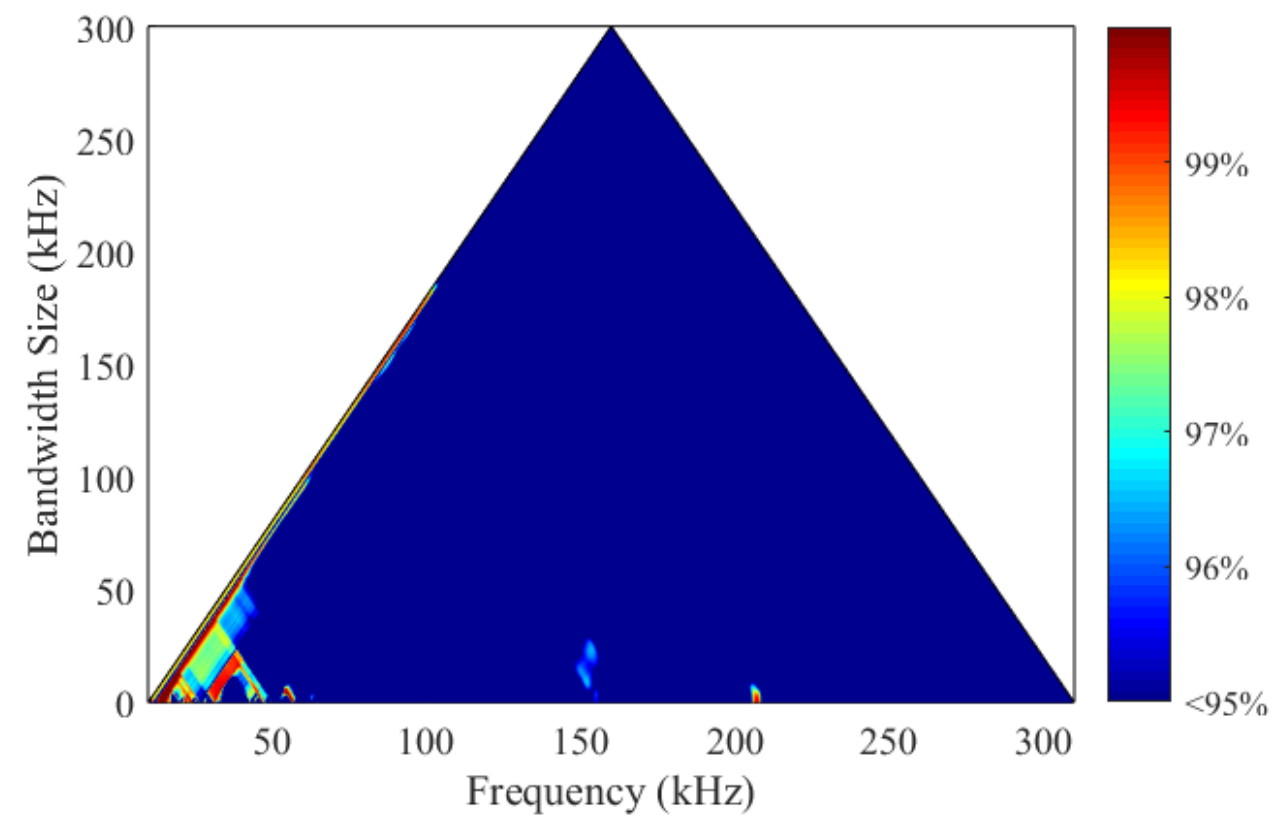

Figure 6. Mapping results for the weakened condition. 


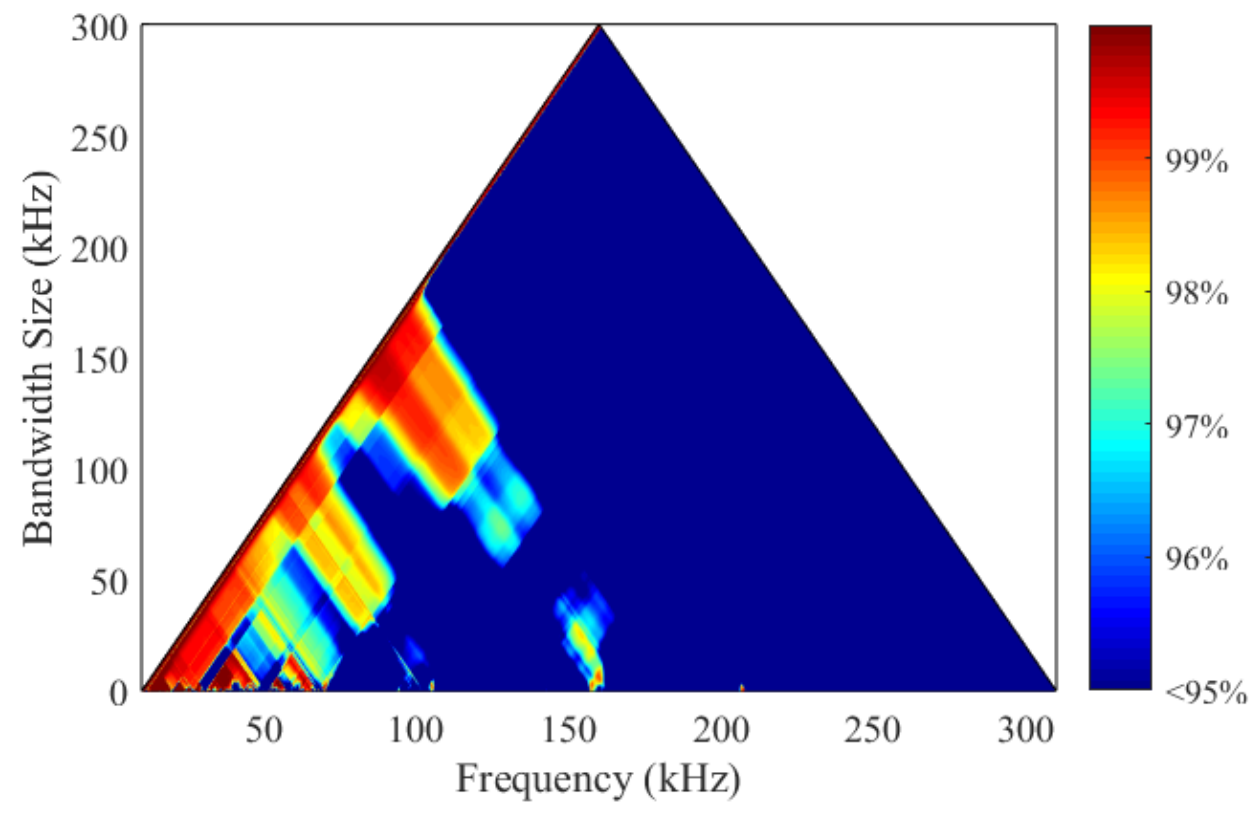

Figure 7. Mapping results for the failed condition.

evaluated using the mapping algorithm. The map is plotted as bandwidth size vs. location of the center of the bandwidth, with the quantitative weights at a given location being the statistical confidence of the difference between the RMSD values for the healthy and weakened condition. Note the two regions which remained undefined (white), as a bandwidth of the given size at the given location would exceed the boundaries of the measured frequency spectrum. Focus is given to regions with statistical confidence greater than $95 \%$. When looking at the map for the weakened implant condition, it is apparent that there are multiple regions in the frequency spectrum where the difference between the healthy and weakened condition is statistically significant. One feature of the map that is particularly interesting is the bright line running diagonally on the left-hand side of the plot. This line is likely caused by the large peaks displayed at $11 \mathrm{kHz}$ which experiences a frequency shift between healthy and unhealthy conditions. Due to the size of these peaks, the RMSD values of the bandwidth which include these peaks are dominated by their contribution. With this said, the regions that show the greatest statistical confidence are at approximately 15,30, and $55 \mathrm{kHz}$. Additionally, there are regions of statistical significance encompassing the peaks at 20,26, and $31 \mathrm{kHz}$ which were mentioned in the previous section. Outside of the lower frequency areas, there is little statistically significant activity, with the exception being a few small regions at 150 and $210 \mathrm{kHz}$.

Moving on to the map in Figure 7 which compares the healthy and failed implants, it is apparent that many more frequency location and bandwidth size options become statistically significant. Additionally, the regions that already displayed a statistically significant difference have increased in confidence, suggesting a greater and more consistent difference between the healthy and failed condition. The largest change occurred in the 50 to $150 \mathrm{kHz}$ range, particularly when a bandwidth size of at least $50 \mathrm{kHz}$ was considered. The best range for detecting failure is still between $10 \mathrm{and} 60 \mathrm{kHz}$. The mapped response at $150 \mathrm{kHz}$ has increased in size and confidence from the weakened comparison. Additionally, the significant region at $210 \mathrm{kHz}$ is still present but does not display a major change from the weakened comparison.

\subsection{Comparison of qualitative and quantitative results}

In order to relate the quantitative results with the features discussed in the qualitative analysis, it is helpful to overlay a few example bandwidths which were identified as statistically significant in the quantitative analysis on top of the impedance data shown in the qualitative analysis. It should be noted that the example bandwidths selected are not exhaustive of all possible bandwidths and serve only to illustrate some of the statistically significant regions. Further, in order to detect weakening or failure, any one of these bands can be selected and evaluated independently. For the weakened damage condition, which is displayed in Figure 8, an example frequency band is selected that encompassed the peak at 11 $\mathrm{kHz}$ as well as the cluster of peaks at 20,26, and $31 \mathrm{kHz}$. Example bands are also selected near $50 \mathrm{kHz}$ as well as at 150 


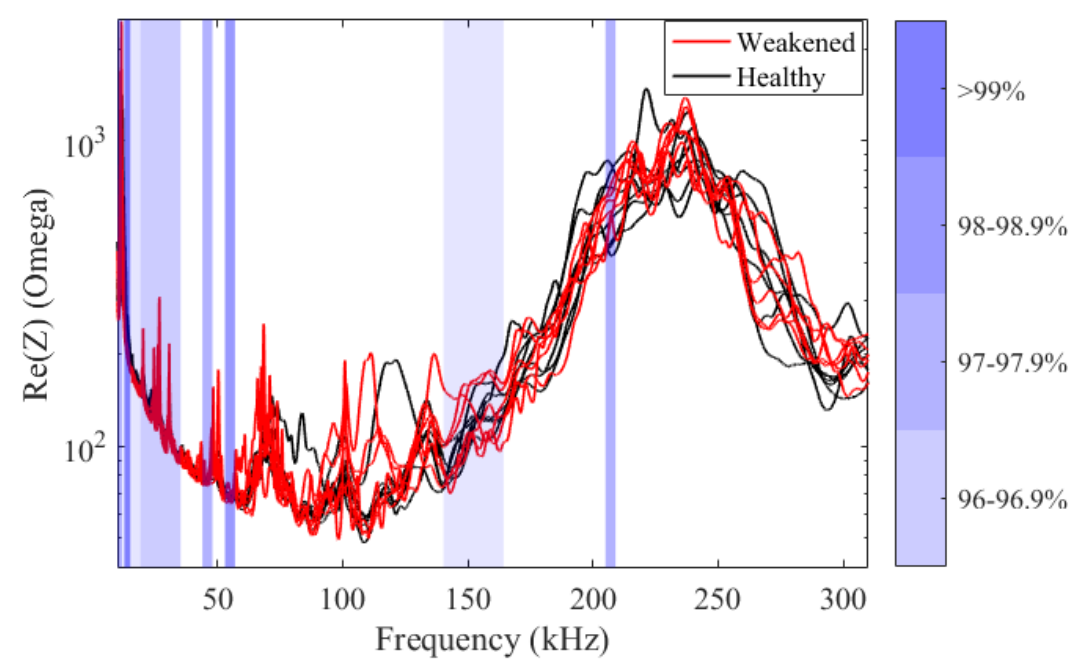

Figure 8. Example bandwidths which are statistically significant in weakening.

and $210 \mathrm{kHz}$. From these example bands, it is still apparent that the area of greatest interest is in the range of $10 \mathrm{kHz}$ to 60 $\mathrm{kHz}$. It should be noted that, although some of the highest impedance values are displayed at above $200 \mathrm{kHz}$, not much of the high frequency range shows consistent difference between the healthy and weakened conditions. It should also be noted that the frequency range of $60 \mathrm{kHz}$ to $80 \mathrm{kHz}$, which was discussed in the qualitative section, did not show a statistically significant bandwidth. This is due to the fact that only one weakened sample showed a difference from the healthy samples, suggesting that it was an outlier rather than a statistically significant trend.

Investigating the failed detection bandwidths in Figure 9, it is immediately apparent that the features of interest discussed in previous sections have increased in statistical confidence. The peaks at 20, 26, and $31 \mathrm{kHz}$ display a much higher confidence during failure when compared to weakening. Additionally, the bands at and around $50 \mathrm{kHz}$ display an increase in statistical significance. It should be noted that the failed damage condition displayed statistically significant bandwidths on the failed map that were much larger in size; however, these bandwidths are not displayed as examples in this figure as they would block out smaller bands. It should be noted additionally that no bandwidth is displayed at $210 \mathrm{kHz}$ as the failed condition map did not display a statistically significant region in this frequency range.

Reflecting on the results presented above, it is apparent that the EMI method is able to effectively detect structural changes between the healthy implant samples and the weakened and failed implants samples. Further, it is apparent that these

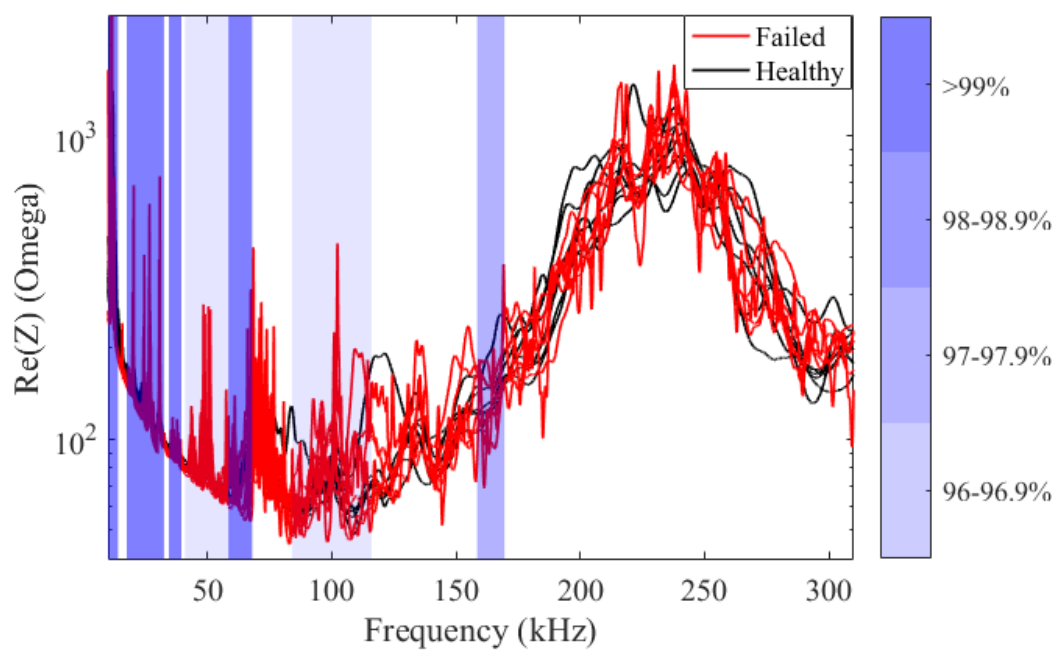

Figure 9. Example bandwidths which are statistically significant in failure. 
differences are statistically significant across multiple samples and can be measured at a variety of frequency bandwidths. From the mapping algorithm, it appears to be that the bandwidths with the highest statistical confidence in the measured frequencies lie roughly between $10 \mathrm{kHz}$ and $75 \mathrm{kHz}$. Additionally, statistical confidence of measured changes increases when going from weakened to failed test samples, suggesting that the differences become larger and more consistent with increased damage. These key findings provide a positive outlook for using EMI based structural health monitoring in detecting mechanical loosening of TKR tibial components.

\section{CONCLUSION}

This study investigated the ability of impedance-based structural health monitoring to detect tibial loosening of a total knee replacement. For this work, experimental test samples involving actual bone cement, synthetic bone (Sawbone), and an actual tibial component were created in order to simulate an implant system. Test samples were created for a healthy implant, an implant which has a weakened cement interface, and an implant which has failed due to loosening. Data was collected for these test setups and qualitatively and quantitatively compared in order to find differences between the healthy and unhealthy samples. An algorithm which mapped statistically significant changes between healthy and unhealthy samples was developed and utilized for quantitative analysis. From the data presented, it is apparent that the EMI method is able to effectively detect both weakening as well as failure in a loosened TKR. It is also apparent from both the qualitative and quantitative data that there is a perceptible difference between an implant which is weakened and one that has failed. This work has also displayed key frequency regions which are useful for identifying both weakened and failed mechanical conditions. Additional work should be done in order to lay the groundwork for a clinical trial of EMI-based SHM of TKRs. A parametric study needs to be performed in order to evaluate whether changing the location of dimensions of the PZT transducer affects the sensitivity of the measurement system. Additionally, investigation into how the measurement system will maintain biocompatibility without compromising damage sensitivity is necessary. This study has the potential to serve as a foundation for those future works as well as laying the long-term groundwork for improved TKR patient outcomes.

\section{ACKNOWLEDGEMENTS}

Research reported in this publication was supported by the National Institute of Arthritis and Musculoskeletal and Skin Diseases of the National Institutes of Health under Award Number R15AR068663. The content is solely the responsibility of the authors and does not necessarily represent the official views of the National Institutes of Health.

\section{REFERENCES}

[1] T. Dixon, M. Shaw, S. Ebrahim et al., "Trends in hip and knee joint replacement: socioeconomic inequalities and projections of need," Annals of Rheumatic Disease, 63(7), 825-30 (2004).

[2] R. B. Bourne, B. M. Chesworth, A. M. Davis et al., "Patient satisfaction after total knee arthroplasty: who is satisfied and who is not?," Clinical Orthopaedics and Related Research, 468(1), 57-63 (2010).

[3] K. J. Bozic, S. M. Kurtz, E. Lau et al., "The epidemiology of revision total knee arthroplasty in the United States," Clinical Orthopaedics and Related Research, 468(1), 45-51 (2010).

[4] P. F. Sharkey, P. M. Lichstein, C. Shen et al., "Why are total knee arthroplasties failing today-has anything changed after 10 years?," The Journal of Arthroplasty, 29(9), 1774-1778 (2014).

[5] M. A. Miller, M. J. Terbush, J. R. Goodheart et al., "Increased initial cement-bone interlock correlates with reduced total knee arthroplasty micro-motion following in vivo service," Journal of Biomechanics, 47(10), 24602466 (2014).

[6] S. Endres, and A. Wilke, "Is cementing technique the cause of early aseptic loosening of the tibial component in total knee arthroplasty? A report of 22 failed tibial components," Orthopedic Reviews, 3(1), 5 (2011).

[7] M. Kozanek, A. Hosseini, F. Liu et al., "Tibiofemoral kinematics and condylar motion during the stance phase of gait," Journal of Biomechanics, 42(12), 1877-84 (2009).

[8] B. Heinlein, F. Graichen, A. Bender et al., "Design, calibration and pre-clinical testing of an instrumented tibial tray," Journal of Biomechanics, 40, S4-S10 (2007). 
[9] M. Safaei, and S. R. Anton, "The effects of dimensional parameters on sensing and energy harvesting of an embedded PZT in a total knee replacement," SPIE Smart Structures and Materials+ Nondestructive Evaluation and Health Monitoring, 97992P-13 (2016).

[10] M. Safaei, R. M. Meneghini, and S. R. Anton, "Energy Harvesting and Sensing With Embedded Piezoelectric Ceramics in Knee Implants," IEEE/ASME Transactions on Mechatronics, 23(2), 864-874 (2018).

[11] K. Worden, C. R. Farrar, G. Manson et al., "The fundamental axioms of structural health monitoring," Proceedings of the Royal Society of London A: Mathematical, Physical and Engineering Sciences, 463(2082), 1639-1664 (2007).

[12] V. Giurgiutiu, [Structural Health Monitoring with Piezoelectric Wafer Active Sensors: with Piezoelectric Wafer Active Sensors] Elsevier, (2007).

[13] I. Pavelko, V. Pavelko, S. Kuznetsov et al., "Bolt-joint structural health monitoring by the method of electromechanical impedance," Aircraft Engineering and Aerospace Technology: An International Journal, 86(3), 207-214 (2014).

[14] E. L. Ribolla, and P. Rizzo, "Modeling the electromechanical impedance technique for the assessment of dental implant stability," Journal of Biomechanics, 48(10), 1713-1720 (2015).

[15] R. I. Ponder, M. Safaei, and S. R. Anton, "Validation of impedance-based structural health monitoring in a simulated biomedical implant system," ASME 2018 Conference on Smart Materials, Adaptive Structures and Intelligent Systems, V002T05A008 (2018).

[16] C. Liang, F. Sun, and C. Rogers, "Coupled electro-mechanical analysis of adaptive material systemsdetermination of the actuator power consumption and system energy transfer," Journal of Intelligent Material Systems and Structures, 8(4), 335-343 (1997).

[17] F. G. Baptista, and J. Vieira Filho, "A new impedance measurement system for PZT-based structural health monitoring," IEEE Transactions on Instrumentation and Measurement, 58(10), 3602-3608 (2009).

[18] A. Arami, J.-R. Delaloye, H. Rouhani et al., "Knee implant loosening detection: a vibration analysis investigation," Annals of Biomedical Engineering, (2017).

[19] C. Farrar, G. Park, H. Sohn et al., "Overview of piezoelectric impedance-based health monitoring and path forward," Shock and Vibration Digest, 35(6), 451-463 (2003).

[20] D. J. Inman, C. R. Farrar, V. L. Junior et al., [Damage Prognosis: for Aerospace, Civil and Mechanical Systems] John Wiley \& Sons, (2005). 